\title{
New Possibilities for In-Situ Electrical Characterisation of Nanosamples at Different Temperatures Combined with Simultaneous TEM Observations
}

\author{
Maria Rudneva, Tatiana Kozlova and Henny W. Zandbergen \\ Delft University of Technology, National Centre for High Resolution Electron Microscopy, Delft, The \\ Netherlands
}

Nanostructures are commonly studied and analysed in the form of end products - synthesized or specifically modified objects on the nanoscale. In that case the physical phenomena taking place during processing or the evolution of the properties of nanostructures often escape the attention they deserve. For example, conventional ex situ studies on the effect of electric current passage through such nanostructures are usually carried out by sequential acquisition of electrical measurement data and microscopic imaging. However, simple interpolation of such data does not always give direct characteristic of the process or of the electrical properties of the sample. For instance, we observed a huge difference in the structure of the polycrystalline platinum $(\mathrm{Pt})$ bridges imaged prior and after application of an electric current, that provoke the particular interest in an in situ investigation of the processes that take place in the bridge during current/voltage stressing.

To understand the details of nanostructural changes in nanodevices due to influence of an electric current in situ imaging should be carried out simultaneously with the electrical measurements. This can be achieved with in situ transmission electron microscopy (TEM).

There are a number of factors that make the real-time observations extremely challenging: sample preparation, contamination, the need for fast scan cameras and difficulties to correlate the applied treatment with the observed changes. Furthermore if the devices to be studied are very small, stray currents can easily destroy the devices. In this study we present the tools and approaches that led to successful in situ real-time observations of current-induced grain growth and electromigration in thin polycrystalline metal nanobridges with TEM.

At first, real-time imaging of changes in shape and microstructure of the sample during application of electrical current was performed. We used a fast scan camera with frame rate up to 25 images per second for video recording. This allows us to correlate features appearing on current-voltage characteristic or changes in resistance of the sample with changes of its nanostructure [1]. For example TEM inspection shows that the drop in the differential resistance that is clearly observed in an I-V curve coincides with thermally induced grain growth in thin polycrystalline films of $\mathrm{Pt}, \mathrm{Pd}$ or $\mathrm{Au}$. The temperature required to initialize the grain growth is estimated numerically and measured directly by combining the use of special MEMS heating devices with TEM observation [2].

Next we recorded in situ electromigration process in $15 \mathrm{~nm}$ thick Pt, Pd and Au bridges of different geometries at room and liquid nitrogen temperatures. The investigation was done in both TEM and scanning-TEM (STEM) modes. The first one gives an overview of the microstructural changes as well as of the process of nanogap formation due to electromigration. The second one allows analysing in situ the mass transfer and thickness evolution along the bridge. The possibility to refill the voids formed during electromigration by changing the sample polarity and thus performing "reversed" electromigration was also studied. 
In the experiments described above a special sample holder built in-house with 8 electric feedthroughs is used for carrying out the electrical measurements. The holder has a cooling option; in such a way both room and low temperature experiments can be done. A chip-like sample with structures patterned onto free standing $\mathrm{SiN}$ membrane is connected to the chip carrier using wire bonding technique. All experiments are performed in a FEI Titan 80-300 microscope operated at 300kV.

The development of this method can potentially yield benefits for nanoscale electronics, measurements on nanowires and graphene nanoribbons, managing of molecular electronic devices and further progress of in situ TEM characterization.

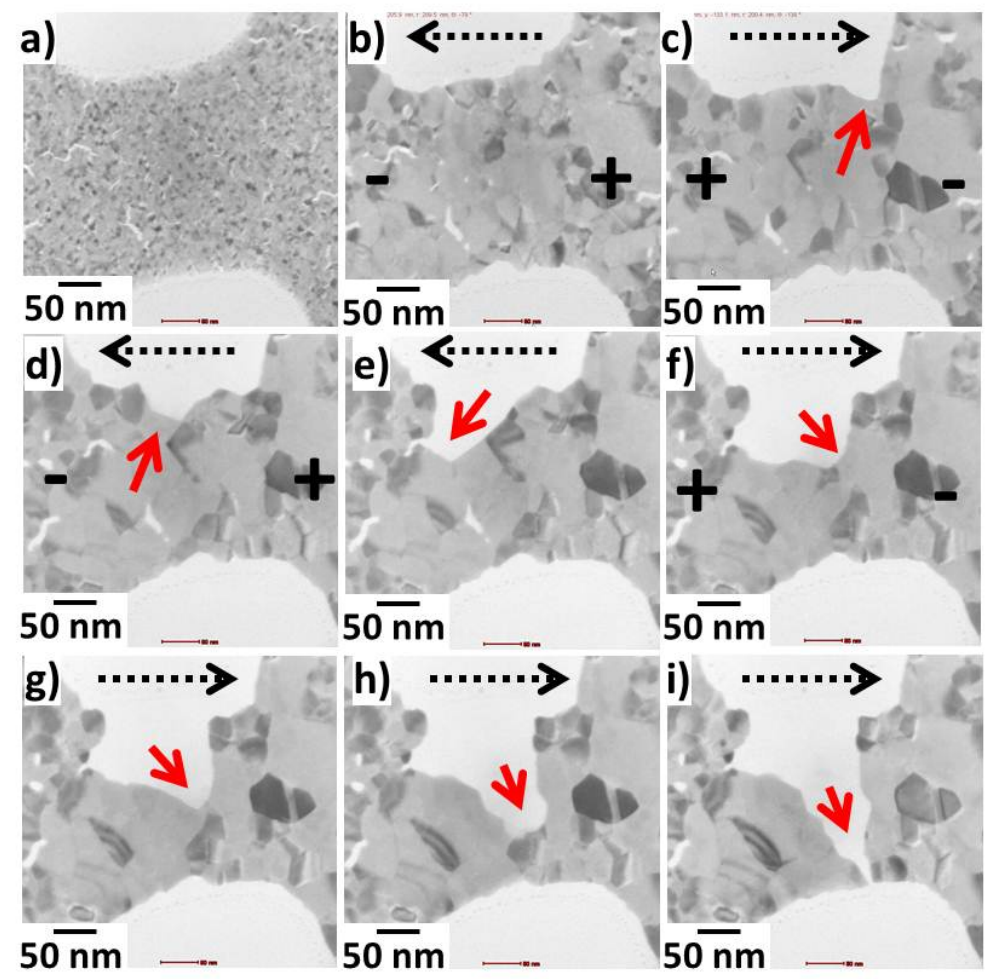

Figure 1. Overview of the reversed electromigration process in $15 \mathrm{~nm}$ thick Pt bridge. (a) The Pt nanobridge as fabricated. Polarity of the sample was changed for a few voltage loops and direction of constriction propagation was followed in-situ. (b) grain growth in the bridge caused by electric current annealing; (c) - (h) constriction propagation in the bridge due to electromigration process; i) the gap is formed close to the cathode side at the end of the electromigration process. The dashed arrows in all images denote the direction of the current

\section{References:}

[1] B. Gao et al, Nanotechnology 22 (2011), 205705

[2] KS McGarrity, Computational Material Science 55 (2011) p.3043-3049

[3] The authors acknowledge funding from the NIMIC consortium (Nano Imaging Under Industrial Conditions) and ERC. 